\title{
Etude Phénotypique des Taurins Ndama du Centre de Recherches Zootechniques de Kolda (Sénégal)
}

\author{
Badji Marc Noël, (Doctorant PhD) \\ Laboratoire National de l'Elevage et de Recherches Vétérinaires (LNERV)/ \\ ISRA, Université Cheikh Anta Diop de Dakar, Département de Biologie \\ Animale \\ Diouf Mame Nahé, PhD \\ Ciss Mamadou, PhD \\ Laboratoire National de l'Elevage et de Recherches Vétérinaires/ ISRA \\ Baldé Amadou Tidiane, MSc \\ Sambe Babacar Souleymane, (Doctorant PhD) \\ Laboratoire National de l'Elevage et de Recherches Vétérinaires (LNERV)/ \\ ISRA, Université Cheikh Anta Diop de Dakar, Département de Biologie \\ Animale \\ Diop Mamadou, PhD \\ Laboratoire National de l'Elevage et de \\ Recherches Vétérinaires (LNERV)/ ISRA \\ Sembène Mbacké, Professeur
}

Université Cheikh Anta Diop de Dakar, Département de Biologie Animale

Doi:10.19044/esj.2020.v16n3p514 ～URL:http://dx.doi.org/10.19044/esj.2020.v16n3p514

Résumé

Au Sud du Sénégal, du fait de la présence de glossines, le taurin Ndama ou Bos taurus taurus est la principale race bovine élevée par les agropasteurs. L'importance indéniable de ces bovins dans la vie socio-économique a conduit à la création du Centre de Recherches zootechniques de Kolda (CRZ-K) et l'initiation d'un programme de sélection à noyau fermé dès 1972 pour améliorer les performances des taurins. L'actuel noyau de sélection ouvert est constitué par des animaux appartenant au CRZ-K et ceux de la Coopérative des Agro-éleveurs Sélectionneurs de la Ndama (CASE-Ndama). L'objectif de cette étude est de caractériser phénotypiquement les taurins Ndama du noyau de sélection. La collecte des données phénotypiques composées de 15 variables qualitatives et 17 quantitatives a été réalisée sur 118 taurins adultes agés d'au moins quatre ans. L'évaluation des performances pondérales a été réalisée grâce à l'analyse des données longitudinales de 1970 bovins durant la période de 1973 à 2016. Les variables qualitatives ont été exprimées en 
pourcentages et pour les variables quantitatives, une analyse descriptive et une comparaison des moyennes ont été faites. Les résultats ont montré que la majorité des animaux présentait la même apparence avec une robe fauve uniforme, des cornes bicolores en lyre, des muqueuses peu pigmentées. Avec un dimorphisme marqué chez les bovins, les animaux adultes ont une hauteur au garrot moyenne de $106,9 \pm 3,5 \mathrm{~cm}$ et un poids moyen de $230,9 \pm 40,6 \mathrm{~kg}$. Les moyennes de la longueur du corps et du périmètre thoracique étaient de $112,0 \pm 7,9 \mathrm{~cm}$ et $150,0 \pm 8,4 \mathrm{~cm}$ respectivement. Cette étude tout en montrant la hausse des mensurations corporelles des animaux par rapport à leurs congénères élevés dans les exploitations rurales souligne la baisse des performances pondérales survenue pendant certaines périodes durant lesquelles le programme a connu des contraintes.

Mots clés : Bos Taurus, Race Ndama, Programme De Sélection, Caractérisation Phénotypique, Performances Pondérales 


\title{
Phenotypic characterization of Ndama taurine cattle of Centre de Recherches Zootechniques of Kolda (Senegal)
}

\author{
Badji Marc Noël, (Doctorant PhD) \\ Laboratoire National de l'Elevage et de Recherches Vétérinaires (LNERV)/ \\ ISRA, Université Cheikh Anta Diop de Dakar, Département de Biologie \\ Animale \\ Diouf Mame Nahé, PhD \\ Ciss Mamadou, PhD \\ Laboratoire National de l'Elevage et de Recherches Vétérinaires/ ISRA
}

Baldé Amadou Tidiane, MSc

Sambe Babacar Souleymane, (Doctorant PhD)

Laboratoire National de l'Elevage et de Recherches Vétérinaires (LNERV)/

ISRA, Université Cheikh Anta Diop de Dakar, Département de Biologie

Animale

Diop Mamadou, PhD

Laboratoire National de l'Elevage et de

Recherches Vétérinaires (LNERV)/ ISRA

Sembène Mbacké, Professeur

Université Cheikh Anta Diop de Dakar, Département de Biologie Animale

\begin{abstract}
In southern Senegal, because of tsetse flies, Ndama taurine or Bos taurus taurus is the main bovine breed raised by farmers. The undeniable importance of these cattle in the socio-economic life led to the creation of the Centre de Recherches Zootechniques of Kolda (CRZ-K) and the initiation of a selection program since 1972 to improve animal productivity. The current open breeding nucleus is composed by animals belonging to the CRZ-K and those of farmers Ndama Agro-Breeders' Cooperative (CASE-Ndama). The aim of this study is to phenotypically characterize Ndama taurins of the selection nucleus. The phenotypic data collected for 15 qualitative and 17 quantitative variables was carried out on 118 adult bulls at least four years old. Longitudinal data from 1970 cattle during the period 1973 to 2016 were used to evaluate weight performances. The results showed that the majority of animals had the same appearance with a uniform fawn color, black and white
\end{abstract}


lyre horns and fairly pigmented mucous membranes. Sexual dimorphism was marked. Adult animals had an average height of $106.9 \pm 3.5 \mathrm{~cm}$ and average weight of $230.9 \pm 40.6 \mathrm{~kg}$. The mean of body length and chest perimeter were $112.0 \pm 7.9 \mathrm{~cm}$ and $150.0 \pm 8.4 \mathrm{~cm}$ respectively. This study showed the increase in body measurements of animals compared to those in rural farms and highlighted the decline in weight performances occurred during certain periods where the program had constraints.

Keywords : Bos Taurus, Ndama Breed, Breeding Program, Phenotypic Characterization, Weight Performance

\section{Introduction}

La race Ndama (Bos taurus taurus) est une des principales races transfrontalières de l'Afrique de l'Ouest. Au Sénégal, ce taurin est élevé essentiellement au Sud du pays en raison de sa trypanotolérance. En effet, dans cette zone humide, infestée de glossines, l'élevage des races trypanosensibles est impossible sans une prophylaxie médicale continue. L'importance de cette race bovine locale dans l'économie des exploitations rurales tient du fait qu'elle assure exclusivement la production laitière locale, une partie de la production carnée et participe fortement à la génération de revenus des populations (MEPA, 2007). Ce constat a depuis longtemps conduit à la création du Centre de Recherches Zootechniques de Kolda (CRZ-K) et l'initiation en 1972 d'un programme d'amélioration génétique par la sélection pour accroitre la productivité du taurin Ndama (Badji, 1973). Ce programme de sélection massale intra-raciale à noyau fermé a prévalu jusqu'en 1991 et des géniteurs sélectionnés ont été diffusés dans les exploitations rurales conformément aux objectifs dudit programme. En 1992, le Système d'Amélioration Génétique à Noyau Ouvert (SAGNO) avait été testé au CRZ$\mathrm{K}$ avec une implication des agropasteurs. En 2010 et 2011, pour remédier au faible effectif du noyau de sélection, des vaches des éleveurs ont été transférées au niveau du noyau. A ce jour, ce noyau de sélection reste encore fermé avec un transfert du matériel génétique sélectionné se faisant uniquement du CRZ-K vers les troupeaux éleveurs.

Ainsi, pendant plusieurs décennies, les animaux qui composent le noyau de sélection se reproduisent exclusivement entre eux et sont isolés de leurs congénères des exploitations rurales et des chocs qu'ils subissent (croisements avec races trypanosensibles, pathologies...). Ce mode de gestion fait que le noyau constitue une population ou souche de taurin Ndama spécifique.

Si de nombreuses études relatives à la santé (Touré et al., 1977 ; Sauveroche et Wagner, 1993 ; Ganyo et al., 2018), la production (Fall et al., 1982 ; Dieye et al., 2003; Kamga-Waladjo et al., 2005 ; Sokouri et al., 2014) 
ont été réalisées sur le taurin Ndama, la diversité génétique des souspopulations ou écotypes de ce taurin dans la zone Sud fait de plus en plus l'objet d'études (Diack 2009 ; Diouf et al., 2011; Camara, 2012 ; Ndiaye, Sawadogo et Sembene, 2015 ; Baldé, 2017). L’objectif de cette étude est de caractériser phénotypiquement les animaux du noyau de sélection du CRZ de Kolda. Elle contribue ainsi à une meilleure connaissance d'un matériel génétique diffusé dans les exploitations rurales.

\section{Matériel et méthodes}

\subsection{Site et matériels de l'étude}

L'étude a été menée au CRZ-K $\left(12^{\circ} 86^{\prime} 62,5^{\prime}\right.$ 'Nord et $14^{\circ} 94^{\prime} 75,4^{\prime \prime}$ 'Ouest) qui s'étend sur 2600 hectares. Ce centre est localisé dans la région de Kolda où le climat est de type soudano-guinéen avec une saison des pluies de 5 mois (de juin à octobre), période à haute incidence des glossines (Touré, 1971 ; Laveissière et Touré, 1982). Le noyau ouvert du programme de sélection est constitué par deux cent vingt-sept (227) taurins Ndama dont cent quarante-cinq (145) animaux représentant $63,5 \%$ de l'effectif total appartenant au CRZ-K et quatre-vingt-deux (82) animaux appartenant aux éleveurs de la Coopérative des Agro-Sélectionneurs de la race Ndama (CASE-Ndama).

Les animaux sont regroupés en quatre (4) lots de reproduction (femelles et géniteur) et un (1) lot de taurillons en pré-testage. Ils sont élevés selon un mode extensif avec une alimentation assurée principalement par les pâturages naturels du CRZ-K. Une complémentation à base de concentrés est distribuée aux jeunes animaux, aux malades et aux femelles gestantes en saison sèche lorsque les pâturages ont un déficit fourrager. La complémentation est généralisée à tous les animaux pendant les périodes de soudure (Avril, Mai). L'abreuvement est à volonté. Les animaux ont été soumis à un suivi sanitaire régulier dont des déparasitages et des vaccinations contre les maladies courantes dans la zone. La filiation, les performances pondérales et reproductives sont enregistrées pour chaque animal identifié par une boucle auriculaire et un numéro marqué au fer à la cuisse.

\subsection{Collecte des données}

Les données ont été collectées en octobre 2016. La collecte de données phénotypiques a concerné cent dix-huit (118) taurins adultes (9 mâles et 109 femelles) agés d'au moins quatre ans. Conformément aux recommandations de la FAO (2012), quinze (15) variables qualitatives et dix-sept (17) quantitatives ont été collectées sur les animaux. Les données ont été enregistrées grâce au Microsoft Excel. Les données relatives aux variables qualitatives ont été décrites après observation directe de l'animal. Il s'agit de : l'aspect de la robe (la couleur du pelage et le type de poil); la présence, la 
forme et la couleur des cornes ; la couleur des muqueuses oculaires, du museau et des sabots ; le port des oreilles ; le profil de la tête et de la ligne du dos ; le niveau de développement du fanon ; la présence ou non de particularités de la robe telles les charbonnures, les bringeures et la raie de mulet (Figure 1).

Les données relatives aux variables quantitatives quant à elles ont été mesurées sur les animaux immobilisés dans un couloir de contention. En fonction de la mensuration (Figure 1), une canne toise, un ruban-mètre et une balance ont été utilisés. Les données suivantes ont été collectées : la longueur de la tête (Lt), la largeur de la tête (lt), la longueur du crâne (Lc), la largeur du crâne (lc), la longueur de la face $(\mathrm{Lf})$, la circonférence du museau $(\mathrm{Cm})$, la longueur des cornes (Lco), la distance intercornes (Di), la longueur des oreilles (Lo), la hauteur au garrot $(\mathrm{Hg})$, la hauteur ou profondeur de poitrine $(\mathrm{Hp})$, la longueur du corps (Lcp) scapulo-ischiale, le périmètre thoracique $(\mathrm{Pt})$, la longueur de la queue (Lq) et le poids (Pds).

Pour l'évaluation des performances pondérales, les données disponibles du suivi pondéral de mille neuf cent soixante-dix (1970) bovins de race Ndama (949 mâles et 1021 femelles) du noyau de sélection du CRZ-K durant la période de 1973 à 2016 ont été analysées. Il s'agit du poids moyen des animaux à différents âge-types : naissance, 3 mois, 6 mois, 9 mois, 12 mois, 18 mois, 24 mois, 30 mois, 36 mois, 42 mois, 48 mois, 54 mois, 60 mois, 66 mois et 72 mois d'âge. A cause de la sortie de certains animaux (réforme ou mortalité), le nombre d'animaux considéré dans cette évaluation est variable pour chaque âge-type considéré.

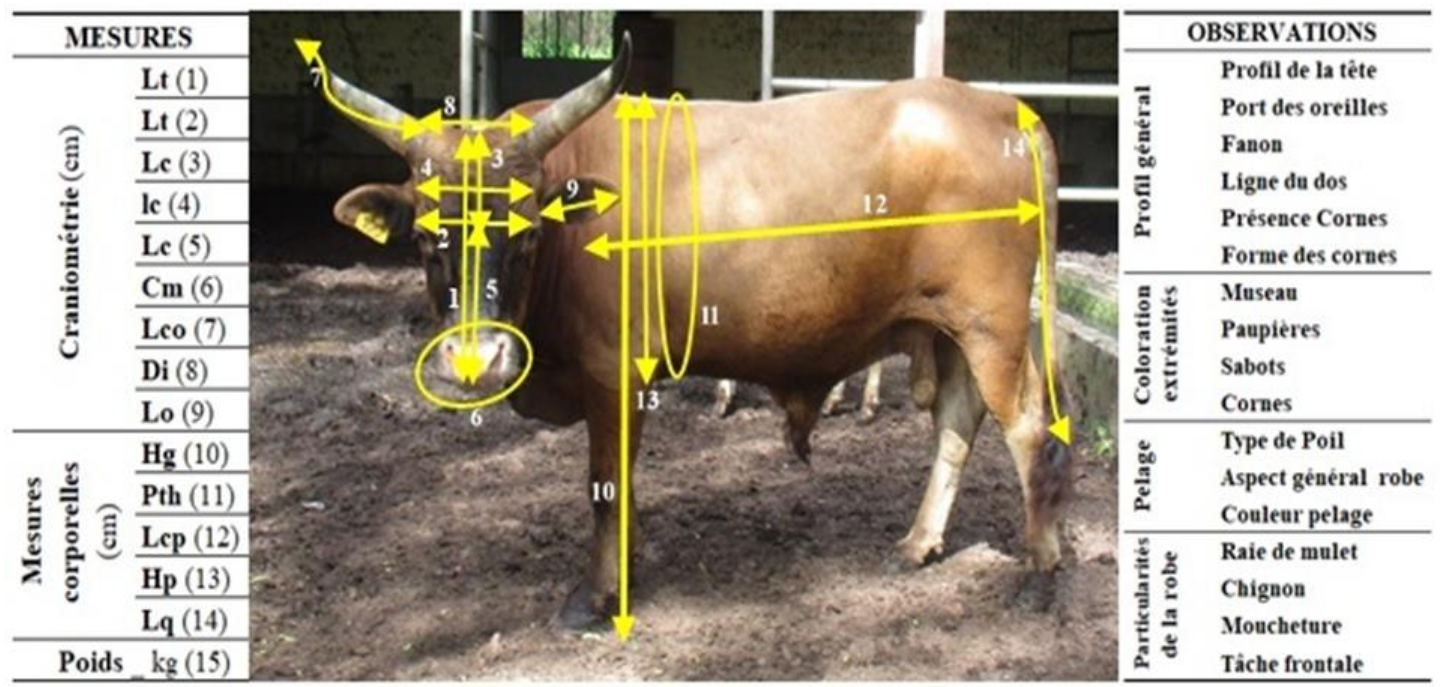

Figure 1. Paramètres pris en compte dans la caractérisation phénotypique du taurin Ndama 


\subsection{Analyses statistiques}

Les variables qualitatives ont été exprimées sous forme de pourcentages. Une analyse descriptive prenant en compte la moyenne, l'écarttype, le minimum et le maximum a été faite sur les variables quantitatives. Des tests de comparaison de moyennes ont été effectués en fonction du sexe et de l'appartenance des animaux. Au préalable les tests de Shapiro-Wilk et de Bartlett permettant de vérifier respectivement la normalité et la similarité des variances entre échantillons avaient été réalisés. Par la suite les tests de comparaisons multiples de Tukey et de Wilcoxon-Mann-Whitney qui permettaient la vérification de la signifiance des différences ont été réalisés. Toutes ces analyses ont été faites grâce au logiciel R version 3.3.1 (R Core Team, 2016). Pour l'ensemble des tests statistiques effectués, le seuil de significativité a été de 5\%.

\section{Resultats}

\subsection{Description Morphologique}

Les taurins Ndama du noyau de sélection du CRZ-K sont des animaux rectilignes et médiolignes. Ils ont un profil de la tête rectiligne, un port des oreilles horizontal et un fanon moyen. Les cornes sont présentes chez tous les animaux et le poil est court (Tableau I, Tableau II). La majorité des animaux présente une robe de couleur fauve uniforme surtout pour les individus appartenant au CRZ-K. Des robes blanches, noires, pie-noires, grises, sables ont été notées chez certains animaux appartenant à la CASE-Ndama (Figure 2). Aussi, les panachures et les charbonnures ont été plus observées chez les individus de la CASE Ndama avec les pourcentages respectifs de 34,09 et 9,09 $\%$. La raie de mulet a été observée sur $50 \%$ des animaux du CRZ-K. Si la majorité des animaux appartenant au CRZ-K a présenté des muqueuses non pigmentées, tel n'est pas le cas de leurs congénères de la CASE-Ndama. Les cornes en lyre ont été décrites chez 47,83\% de l'échantillon d'étude. Elles ont été observées sur 64,38\% des animaux appartenant au CRZ-K. Les animaux ayant des cornes en coupe et en croissant représentent respectivement 27,83\% et $19,13 \%$ de l'échantillon d'étude. Les cornes bicolores noir et blanc ont été observées sur la majorité des animaux étudiés. 
Tableau I. Caractères morphologiques de la tête des taurins Ndama adultes du Centre de Recherches Zootechniques de Kolda

\begin{tabular}{|c|c|c|c|c|}
\hline Variables & Modalités & $\begin{array}{r}\mathrm{CN}(\mathrm{n}=44) \\
(\%)\end{array}$ & $\begin{array}{r}\text { CRZ-K (n=74) } \\
(\%)\end{array}$ & $\begin{array}{r}\text { Total }(\mathrm{n}=118) \\
(\%)\end{array}$ \\
\hline Profil de la tête & Rectiligne & 100 & 100 & 100 \\
\hline Port des oreilles & Horizontal & 100 & 100 & 100 \\
\hline Cornes & Présentes & 100 & 100 & 100 \\
\hline \multirow{7}{*}{ Forme des cornes } & Coupe & 40,48 & 20,55 & 27,83 \\
\hline & Couronne & 4,76 & 0 & 1,74 \\
\hline & Croissant & 35,71 & 9,59 & 19,13 \\
\hline & En arrière & 0 & 2,74 & 1,74 \\
\hline & Asymétrique & 0 & 1,37 & 0,87 \\
\hline & Lyre & 19,05 & 64,38 & 47,83 \\
\hline & Roue & 0 & 1,37 & 0,87 \\
\hline \multirow{3}{*}{$\begin{array}{l}\text { Couleur des } \\
\text { cornes }\end{array}$} & Bicolores & 93,18 & 93,24 & 93,22 \\
\hline & Brunes & 2,27 & 6,76 & 5,08 \\
\hline & Noires & 4,55 & 0 & 1,69 \\
\hline \multirow{2}{*}{ Museau } & Pigmenté & 50 & 8,11 & 23,73 \\
\hline & Non Pigmenté & 50 & 91,89 & 76,27 \\
\hline \multirow{2}{*}{ Paupières } & Pigmentées & 50 & 5,48 & 22,73 \\
\hline & Non Pigmentées & 50 & 94,52 & 76,27 \\
\hline \multirow{2}{*}{ Chignon } & Présent & 4,55 & 9,46 & 7,63 \\
\hline & Absent & 95,45 & 90,54 & 92,37 \\
\hline \multirow{2}{*}{ Moucheture } & Présente & 6,98 & 0 & 2,61 \\
\hline & Absente & 93,02 & 100 & 97,39 \\
\hline \multirow{2}{*}{ Tâche frontale } & Présente & 0 & 6,85 & 4,27 \\
\hline & Absente & 100 & 93,15 & 95,73 \\
\hline
\end{tabular}

n : nombre d'animaux ; CN : CASE-Ndama ; CRZ-K : Centre de Recherches Zootechniques de Kolda. 
Tableau II. Caractères morphologiques du corps des taurins Ndama adultes du Centre de Recherches Zootechniques de Kolda

\begin{tabular}{|c|c|c|c|c|}
\hline Variables & Modalités & $\begin{array}{r}\mathrm{CN}(\mathrm{n}=44) \\
(\%)\end{array}$ & $\begin{array}{r}\text { CRZ-K (n=74) } \\
(\%)\end{array}$ & $\begin{array}{r}\text { Total }(\mathrm{n}=118) \\
(\%)\end{array}$ \\
\hline Fanon & Moyen & 100 & 100 & 100 \\
\hline Ligne du dos & Rectiligne & 100 & 100 & 100 \\
\hline \multirow{5}{*}{ Robe } & Uniforme & 50 & 87,84 & 73,73 \\
\hline & Panachée & 34,09 & 4,05 & 15,25 \\
\hline & Charbonnée & 9,09 & 6,76 & 7,63 \\
\hline & Charb-Panach & 4,54 & 1,35 & 2,54 \\
\hline & Pie & 2,27 & 0 & 0,85 \\
\hline \multirow{9}{*}{ Couleur du pelage } & Fauve & 65,91 & 97,22 & 85,34 \\
\hline & Fauve rouge & 6,82 & 27,78 & 19,83 \\
\hline & Fauve roux & 13,64 & 30,56 & 24,14 \\
\hline & Fauve froment & 45,45 & 38,89 & 41,38 \\
\hline & Sable & 2,27 & 1,39 & 1,72 \\
\hline & Grise & 2,27 & 0 & 0,86 \\
\hline & Blanche & 13,64 & 1,39 & 6,03 \\
\hline & Noire & 13,64 & 0 & 5,17 \\
\hline & Pie-noire & 2,27 & 0 & 0,86 \\
\hline Type de poil & Court & 100 & 100 & 100 \\
\hline \multirow{2}{*}{ Raie de mulet } & Présente & 13,51 & 50 & 36,36 \\
\hline & Absente & 86,49 & 50 & 63,64 \\
\hline \multirow{2}{*}{ Sabot } & Pigmentés & 92,31 & 68,57 & 77,06 \\
\hline & Non Pigmentés & 7,69 & 31,43 & 22,94 \\
\hline
\end{tabular}

$\mathrm{n}$ : nombre d'animaux ; CN : CASE-Ndama ; CRZ-K : Centre de Recherches Zootechniques de Kolda ; Charb-Panach : charbonnée et panachée.

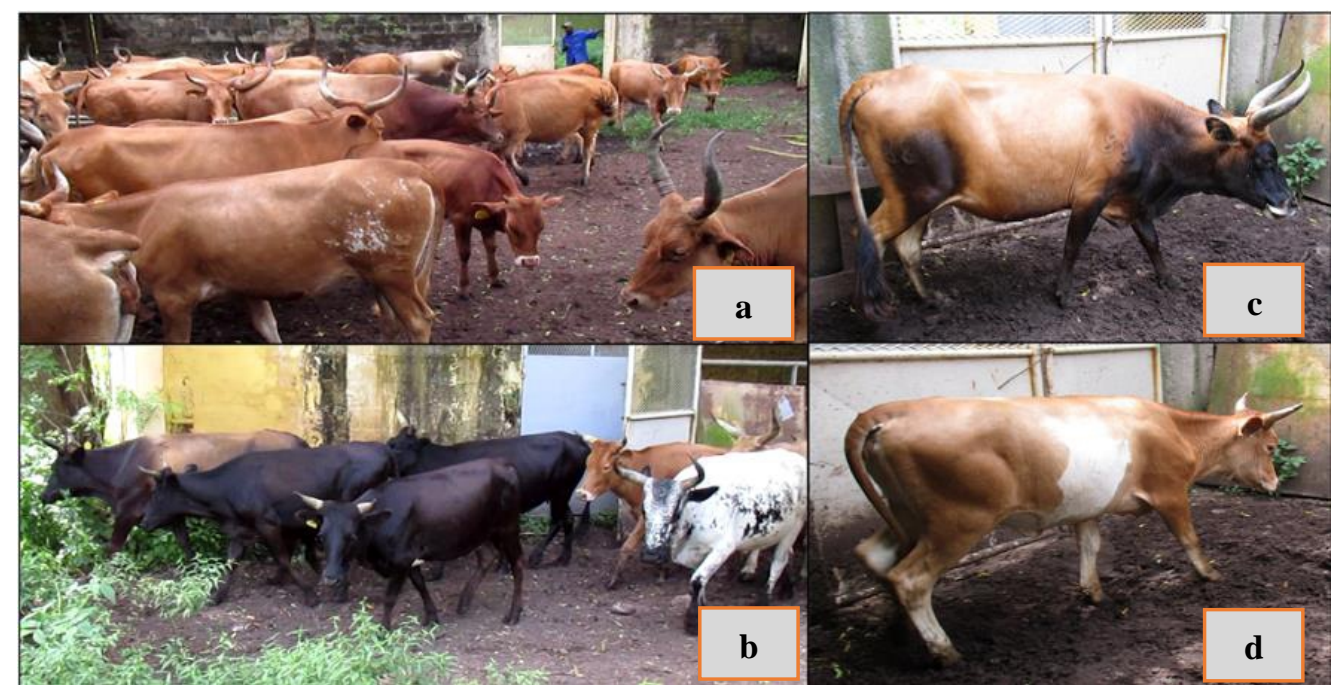

Figure 2. Caractères morphologiques des taurins Ndama du Centre de Recherches

Zootechniques de Kolda. (a) Animaux appartenant au CRZ-K; b) animaux appartenant à la CASE-Ndama; (c) vache présentant des charbonnures $(c)$ et $(d)$ vache présentant une panachure. 


\subsection{Description biométrique}

\subsubsection{Mensurations corporelles}

Les taurins Ndama adultes du noyau de sélection ont une hauteur au garrot $(\mathrm{Hg})$ moyenne de 106,9 $\pm 3,5 \mathrm{~cm}$ et un poids moyen (Pds) de 230,9 \pm 40,6 kg. La longueur du corps ( $\mathrm{Lcp}$ ) et le périmètre thoracique $(\mathrm{Pt})$ sont respectivement de $112,0 \pm 7,9 \mathrm{~cm}$ et $150,0 \pm 8,4 \mathrm{~cm}$. La queue $(\mathrm{Lq})$ mesure en moyenne $80,9 \pm 7,8 \mathrm{~cm}$. Le dimorphisme sexuel est marqué avec des mensurations moyennes pour le taureau de $108,5 \pm 5,9 \mathrm{~cm}$ pour la hauteur au garrot, $123,0 \pm 8,7 \mathrm{~cm}$ et $160,7 \pm 13,5 \mathrm{~cm}$ respectivement pour la longueur $\mathrm{du}$ corps et le périmètre thoracique. Le poids moyen du taureau est de 315,7 \pm $63,8 \mathrm{~kg}$ avec un minimum de $231 \mathrm{~kg}$ et un maximum de $392 \mathrm{~kg}$. Pour l'ensemble des vaches du noyau de sélection, un poids moyen de $223,9 \mathrm{~kg}$ a été rapporté avec des valeurs extrêmes de 130 et $289 \mathrm{~kg}$ (Tableau III). Une hauteur au garrot moyenne de $107,0 \pm 3,4 \mathrm{~cm}$ a été rapportée pour les vaches appartenant au CRZ-K et 106,4 $\pm 3,1 \mathrm{~cm}$ pour celles de la CN. Les moyennes de la longueur du corps et du périmètre thoracique sont respectivement de $111,6 \pm 8,0 \mathrm{~cm}$ et $149,5 \pm 7,8 \mathrm{~cm}$ pour les femelles du CRZ-K et $110,5 \pm 5,9$ $\mathrm{cm}$ et $148,7 \pm 6,8 \mathrm{~cm}$ pour celles de la $\mathrm{CN}$.

Tableau III. Mensurations corporelles et poids des taurins Ndama adultes du Centre de Recherches Zootechniques de Kolda

\begin{tabular}{|c|c|c|c|c|c|c|}
\hline \multirow{2}{*}{\multicolumn{2}{|c|}{ Mensurations }} & & \multirow{2}{*}{$\begin{array}{c}\text { Total } \\
(\mathrm{N}=118)\end{array}$} & \multicolumn{3}{|c|}{ Sexe et appartenance } \\
\hline & & & & $\begin{array}{l}\text { Mâles } \\
(\mathrm{N}=9)\end{array}$ & $\begin{array}{c}\text { Femelles CRZ-K } \\
(\mathbf{N}=66)\end{array}$ & $\begin{array}{c}\text { Femelles CN } \\
(N=43)\end{array}$ \\
\hline \multirow{9}{*}{ 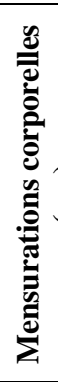 } & Haut & Extrêmes & $97,5-117,8$ & $101,1-117,8$ & $97,5-113,4$ & $100,4-113,2$ \\
\hline & Hau & Moy. \pm Et & $106,9 \pm 3,5$ & $108,5 \pm 5,9$ & $107,0 \pm 3,4$ a & $106,4 \pm 3,1^{a}$ \\
\hline & -ique (Pt) & Extrêmes & $129,0-177,8$ & $139,2-177,8$ & $129,0-170,3$ & $133,9-163,7$ \\
\hline & oracique (It) & Moy. \pm Et & $150,0 \pm 8,4$ & $160,7 \pm 13,5^{*}$ & $149,5 \pm 7,8^{\text {a }}$ & $148,7 \pm 6,8^{a}$ \\
\hline & Longueur du corps (Lcp) & Extrêmes & 36,8 & $113,5-136,8$ & $94,0-133,9$ & $96,5-121,7$ \\
\hline & & Extrêmes & $\begin{array}{l}112,0 \pm 1,9 \\
47,4-66,5\end{array}$ & $\begin{array}{c}123,0 \pm 8,1 \cdots \\
55,4-64,4\end{array}$ & $\begin{array}{l}111,6 \pm 8,0 \\
47,4-66,5\end{array}$ & $\begin{array}{r}110,5 \\
51,0\end{array}$ \\
\hline & Profondeur de poitrine $(\mathrm{Hp})$ & Moy. \pm Et & $56,4 \pm 3,4$ & $59,2 \pm 3,0^{*}$ & $56,5 \pm 3,6^{\mathrm{a}}$ & 55,6 \\
\hline & Lon: & Extrêmes & $23,8-96,5$ & $74,8-96,5$ & $67,4-93,2$ & $23,8-88,4$ \\
\hline & Longueu & Moy. \pm Et & $80,9 \pm 7,8$ & $84,7 \pm 8,3$ & $81,9 \pm 5,34^{\text {a }}$ & $78,8 \pm 10,1^{\mathrm{a}}$ \\
\hline \multirow{2}{*}{\multicolumn{2}{|c|}{ Poids (Pds) - Kg }} & Extrêmes & $130,0-392,0$ & $231,0-392,0$ & $130,0-283,0$ & $166,0-289,0$ \\
\hline & & Moy. \pm Et & $230,9 \pm 40,6$ & $315,7 \pm 63,8 *$ & $225,1 \pm 29,6^{\mathrm{a}}$ & $222,0 \pm 28,2^{\mathrm{a}}$ \\
\hline
\end{tabular}

a, b sur la même ligne, les valeurs non affectées de la même lettre sont statistiquement différentes ( $\mathrm{p}$-value $<0,05) ; *$ différence significative entre mâles et femelles ; Moy $=$ Moyenne $; \mathrm{Et}=$ Ecart-type $; \mathrm{CRZ}-\mathrm{K}:$ animaux appartenant au CRZ de Kolda ; CN : animaux appartenant à la CASE-Ndama

\subsubsection{Mensurations de la tête}

Les moyennes de la longueur (Lt) et de la largeur (lt) de la tête sont de $41,7 \pm 2,7 \mathrm{~cm}$ et $20,0 \pm 1,4 \mathrm{~cm}$ respectivement. Les moyennes enregistrées pour les autres paramètres se présentent comme suit : $16,8 \pm 1,8 \mathrm{~cm}$ pour la longueur des oreilles (Lo), 39,1 $\pm 2,4 \mathrm{~cm}$ pour la circonférence du museau 
$(\mathrm{Cm}), 37,8 \pm 6,7 \mathrm{~cm}$ pour la longueur des cornes (Lco) et 13,3 $\pm 1,1 \mathrm{~cm}$ l'espace entre les cornes (Di) (Tableau IV).

Tableau IV. Mensurations de la tête des taurins Ndama adultes du Centre de Recherches Zootechniques de Kolda

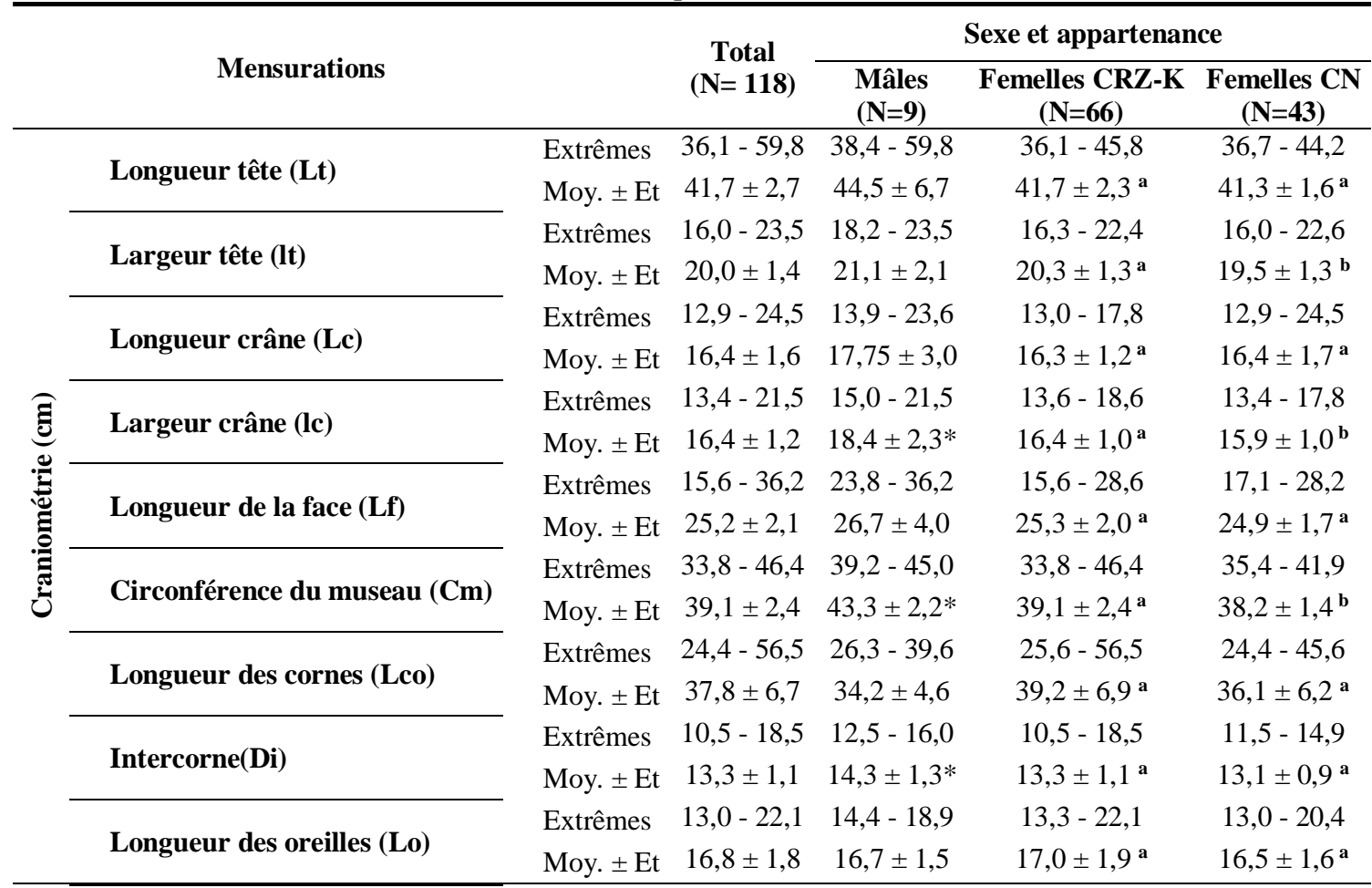

a, b sur la même ligne, les valeurs non affectées de la même lettre sont statistiquement différentes ( $\mathrm{p}$-value $<0,05) ; *$ différence significative entre mâles et femelles ; Moy = Moyenne ; Et = Ecart-type ; CRZ-K : animaux appartenant au CRZ de Kolda ; CN : animaux appartenant à la CASE-Ndama

A l'âge de quatre (4) ans, aucune différence significative n'a été observée entre les classes d'âge des femelles pour les mesures de la tête. Il en est de même à l'âge de cinq (5) ans pour la hauteur au garrot, le périmètre thoracique, la longueur du corps, la profondeur de thorax ainsi que le poids. A partir de 5 ans d'âge, seules les cornes continuent à croître chez les individus. Les femelles du CRZ-K ont en moyenne une légère supériorité par rapport à leurs congénères de la $\mathrm{CN}$ pour l'ensemble des mesures de la tête, du corps et du poids, à l'exception de la longueur du crâne, avec des différences significatives notées pour les largeurs de la tête et du crâne et la circonférence du museau.

Le dimorphisme sexuel est marqué avec une supériorité de l'ensemble des mesures des mâles par rapport aux femelles à l'exception de la longueur 
Des maximums de poids de $377 \mathrm{~kg}$ et $485 \mathrm{~kg}$ ont été enregistrés respectivement à 66 mois d'âge pour les femelles en 1974 et à 72 mois d'âge pour les mâles en 1976 (Figure 3).
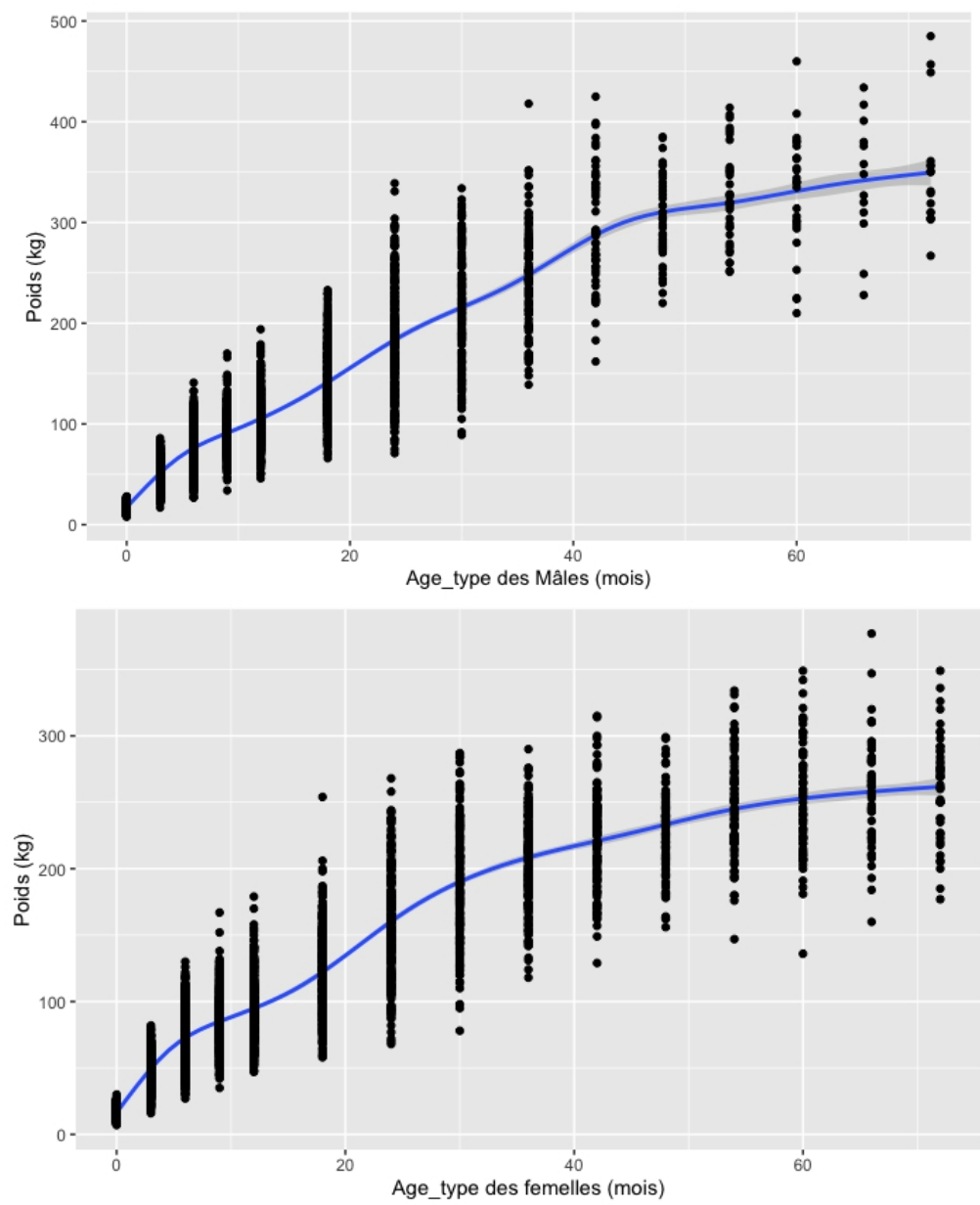

Figure 3 . Evolution pondérale des taurins Ndama du Centre de Recherches Zootechniques de Kolda 
Durant la période 1973-2016, des différences de performances pondérales aux différents âge-types ont été notées selon les décennies. Ainsi, durant la période $1973-1980$, les poids moyens de $17,52 \pm 3,33 \mathrm{~kg}, 81,55 \pm 20,45 \mathrm{~kg}$ et 109,37 $\pm 24,53 \mathrm{~kg}$ ont été enregistrés respectivement à la naissance, à 6 mois et à 12 mois d'âge. Les performances pondérales des animaux durant les périodes 1973-1980 et 1981-1990 sont significativement supérieures à celles de la période 2011-2016 (Figure 4). Durant cette dernière, les poids moyens de $15,72 \pm 1,3 \mathrm{~kg}, 53,28 \pm 21,03 \mathrm{~kg}$ et $72,0 \pm 16,86 \mathrm{~kg}$ ont été enregistrés respectivement à la naissance, à 6 mois et à 12 mois d'âge. Il ressort de l'analyse des performances des animaux qu'avec le mode de conduite en vigueur au CRZ-K durant la période 2011-2016, la croissance des animaux a été lente comme en témoigne la lente évolution pondérale aux différents âgestypes (Figure 4).
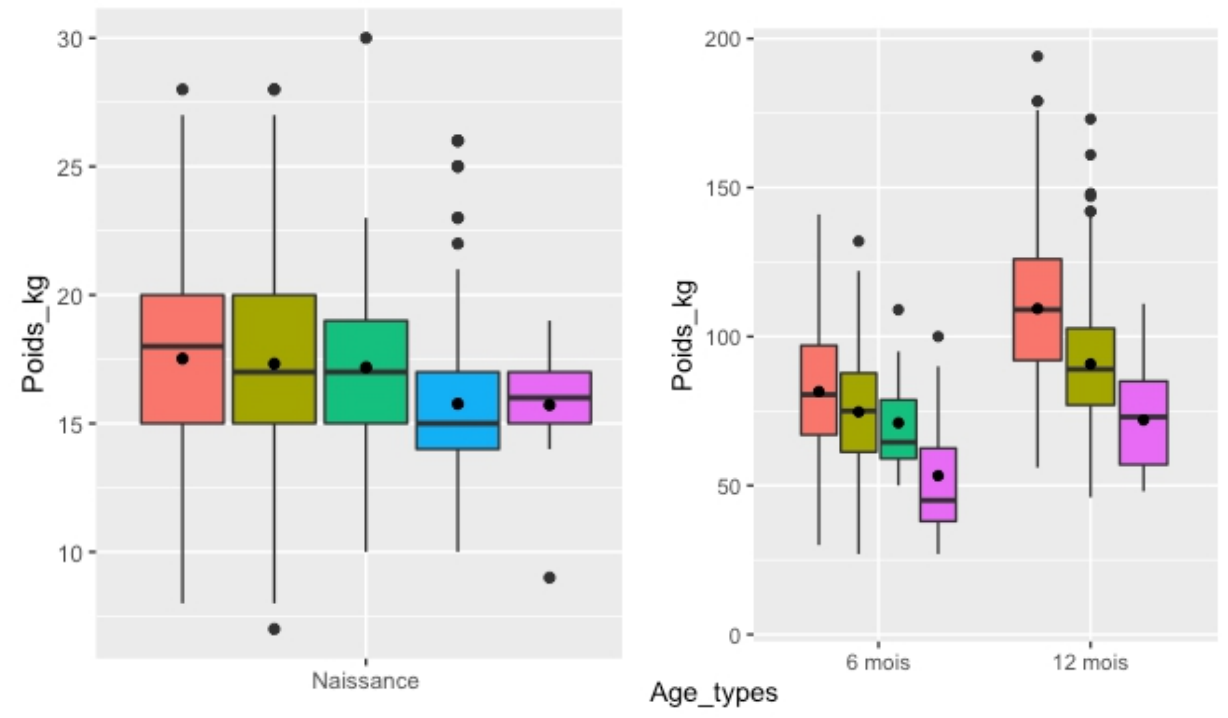

Periodes

Age_types

Figure 4. Evolution comparative des poids moyens des taurins Ndama du Centre de Recherches Zootechniques de Kolda durant la période de 1973 à 2016.

\section{Discussion}

Le taurin Ndama du fait de sa trypanotolérance a fait l'objet de beaucoup d'études dans plusieurs pays (Doutressoulle, 1947 ; Pagot et Delaine, 1959 ; Epstein, 1971 ; Fall et al.,1982 ; Planchenault et al., 1984 ; Diop et al., 1993 ; Sokouri et al., 2007 ; Khang'Mate et al., 2000 ; Akouango et al., 2010 ; Okouyi et al., 2014 ; Ndiaye, 2015). Chez les bovins Ndama qui constituent le noyau de sélection du CRZ-K, la robe fauve uniforme est dominante car elle a été prise en compte dans les critères de sélection (Badji, 1973, Gueye et al., 1979 ; Camara, 2012 ; Wane et al., 2017). Cette coloration appréciée des éleveurs qui l'associe à la trypanotolérance de l'animal ne 
constitue pas en fait une preuve de ce caractère selon Touré et al. (1977), Diaïté (1980). Les robes claires sont associées à l'introgression de sang zébu (Gueye et al., 1979). Outre, la couleur de la robe, les caractères morphologiques des taurins Ndama du CRZ-K sont similaires à ceux décrits par Ndiaye (2015), Diack (2009) et Baldé (2017) dans la zone de Kédougou, au Centre de Conservation, de Multiplication et de Diffusion du Bétail Ruminant Endémique (CCMD/BRE) de Madina-Diassa au sud du Mali (Coulibaly et al., 2014), en Guinée (Kamga-Waladjo et al., 2005) et au Congo (Akouango et al., 2010). Comme rapporté par Sambe et al. (2019), l'apparence similaire des animaux du noyau de sélection est une conséquence de la pression de sélection et de l'application du schéma de sélection à noyau fermé. Il faut souligner que la mise en œuvre du schéma de sélection à noyau ouvert au CRZ-K date des années 1990 et bien que le critère « robe fauve » n'ait été pris en compte lors du choix des vaches transférées dans le noyau, aucun mâle né de ces vaches n'a encore été sélectionné comme géniteur au CRZ-K. Tout comme les animaux appartenant à la CASE Ndama, la pigmentation des phanères et muqueuses, la diversité du type de cornage, de la coloration et les particularités de la robe ont été rapportées sur les taurins Ndama des régions Sud du Sénégal (Gueye et al., 1979 ; Diack, 2009 ; Baldé, 2017), au nordouest et au centre de la Côte d'Ivoire (N'goran et al., 2018).

Considérant les mensurations et certains caractères morphologiques, trois sous-populations ou écotypes de taurin Ndama que sont la grande, moyenne, petite Ndama ont été décrites au Sud du Sénégal. En effet, Diaïté (1980) a indiqué que la hauteur au garrot de la grande Ndama variait entre 108 et $129 \mathrm{~cm}$. Les études de Diack (2009) dans les exploitations rurales ont rapporté une hauteur au garrot de $108,7 \mathrm{~cm}$ pour la grande Ndama, 104,5 cm pour la moyenne et de $100,7 \mathrm{~cm}$ pour la petite Ndama. Les récents travaux de Baldé (2017) ont confirmé la notion de sous-populations de Ndama en rapportant une hauteur moyenne au garrot de $97,93 \pm 5,16 \mathrm{~cm}$ pour le taurin Ndama de la région de Kédougou, 102,95 $\pm 3,25 \mathrm{~cm}$ et 104,21 $13,87 \mathrm{~cm}$ respectivement pour les régions de Kolda et Sédhiou. Notre étude axée sur le noyau de sélection du CRZ-K en rapportant une hauteur au garrot de 110,30 \pm $5,83 \mathrm{~cm}$ pour les mâles et $107,67 \pm 3,12 \mathrm{~cm}$ et $106,87 \pm 3,06 \mathrm{~cm}$ respectivement pour les femelles appartenant au CRZ-K et à la CASE-Ndama met en exergue le gain en taille du taurin Ndama issu de la sélection par rapport à ses congénères des exploitations rurales. En effet, cette valeur moyenne de la hauteur au garrot des animaux appartenant au CRZ-K est similaire à celle de la grande Ndama rapportée par Baldé (2017). Pourtant du point de vue génétique, les résultats préliminaires de Diouf et al. (2012) ont montré que les animaux du CRZ-K sont plus proches des taurins de la région de Kédougou qui ont moins d'introgression de sang zébu. Ceci illustre le progrès génétique du programme de sélection réalisé en termes de gain de taille chez les 
individus sélectionnés et la préservation de leur trait adaptatif qu'est la trypanotolérance. Dans ce sens, la caractérisation moléculaire les vaches de la CASE-Ndama à transférer dans le noyau de sélection du CRZ-K serait nécessaire pour la préservation du trait adaptation chez les futures générations. Aussi, la corrélation positive entre la longueur du corps de l'animal, le périmètre thoracique et le poids fait que ces mensurations peuvent être informatives dans la collecte des performances des individus et surtout lors des dépistages en milieu éleveur.

Des études récentes sur les taurins Ndama de la Côte d'Ivoire et du Congo ont montré une hauteur au garrot et un poids supérieur aux mensurations des animaux de notre étude. En effet, les travaux d'Akouango et al. (2010) ont montré que les taurins Ndama du Congo bien qu'ayant une hauteur au garrot supérieure $(111,8 \pm 0,9$ et 115,3 $\pm 1,1 \mathrm{~cm}$ respectivement pour les femelles et les mâles) à celle des taurins Ndama du Sénégal, présentent une longueur du corps inférieure $(98,2 \pm 0,3$ pour les femelles et 103,8 \pm 0,9 pour les mâles). En Côte d'Ivoire, Coulomb (1976) indiquait une hauteur au garrot de $116 \pm 1,6 \mathrm{~cm}$ chez le mâle et $113,6 \pm 0,8 \mathrm{~cm}$ chez la femelle. L'étude plus récente réalisée en milieu éleveur de N'goran et al. (2018) a mentionné des moyennes de $110,59 \pm 7,33 \mathrm{~cm}$ pour la hauteur au garrot, $121,77 \pm 12,61$ et $150,92 \pm 14,56 \mathrm{~cm}$ respectivement pour la longueur du corps $(\mathrm{Lc})$ et le périmètre thoracique $(\mathrm{Pt})$.

Concernant les performances pondérales, sur les animaux du noyau du CRZ-K, Fall et al. (1982) ont rapporté un poids moyen de $200 \mathrm{~kg}$ à 24 mois au niveau des taurillons en testage qui avaient une hauteur moyenne au garrot de $111 \pm 2,58 \mathrm{~cm}$ selon Gueye et al., (1980). Sur les performances pondérales, les travaux d'Aboagye (2002) au Ghana ont rapporté que l'héritabilité du poids à la naissance chez le taurin Ndama est de 0,45. Camara (2012) a indiqué des corrélations génétiques de 0,25 entre le poids à la naissance et le poids à 12 mois et 0,73 entre le poids à la naissance et le poids à 6 mois. Ces résultats sont similaires à ceux de Fall et al. (1982), Mackinnon et al. (1991) et Bosso (2006). En milieu traditionnel, un poids à la naissance de $14,05 \mathrm{~kg}$ a été enregistré pour le taurin Ndama (Rapport CRZ- Kolda, 1984). Au CRZ-K, Fall et al. (1982) ont noté un poids de $19,1 \mathrm{~kg}$ pour les animaux du noyau de sélection contre $17,6 \mathrm{~kg}$ pour ceux du troupeau fondateur. L'analyse de l'ensemble des performances pondérales des taurins du noyau de sélection de 1973 à 2016 montre une baisse du poids à la naissance atteignant 15,57 kg pour les mâles et 15,44 kg pour les femelles entre 2011 et 2016. Ces valeurs bien que faibles, sont toutefois supérieures aux poids à la naissance enregistrés au Mali et en Guinée. En effet, Coulibaly et al. (2014) ont rapporté un poids à la naissance de $15 \mathrm{~kg}$ au Ranch de Madina-Diassa (Mali) contre 10 et $12 \mathrm{~kg}$ en milieu éleveur. En Guinée, Kamga-Waladjo et al. (2005) ont indiqué un poids à la naissance de $14,9 \pm 1,8 \mathrm{~kg}$ et un poids compris entre 10 et $12 \mathrm{~kg}$ 
avait été rapporté pour le taurin Ndama de type Foula de la Guinée-Bissau (FAO, 1985).

Wane et al. (2017) avaient rapporté en plus de la baisse du poids à la naissance, un allongement de l'intervalle vêlage-vêlage chez les femelles et Sambe et al. (2019) soulignaient les effets négatifs du manque de financement sur l'efficience des programmes de sélection. Ce qui pourrait expliquer la baisse des performances pondérales des animaux du noyau de sélection notée durant certaines décennies.

L'ouverture du noyau de sélection qui s'est traduite par le transfert des vaches des éleveurs dans le noyau est bénéfique à double titre. En effet, en sus d'augmenter l'effectif des vaches du noyau de sélection, les éleveurs en tant que bénéficiaires directs sont sensibilisés sur l'intérêt de la préservation de cette ressource zoogénétique locale et son utilisation durable (Diop et al., 1993 ; Sissokho et al., 2010 ; PROGEBE ILRI, 2011, Camara et al., 2019a ; Camara et al., 2019b). Une plus large diffusion du progrès génétique pourrait être réalisée par le programme avec la pratique de l'insémination artificielle. La caractérisation génétique des bovins du CRZ-K est tout aussi nécessaire afin de connaitre leur statut qui serait utile dans les programmes de conservation car ces taurins ont été élevés en marge des brassages avec les races trypanosensibles.

\section{Conclusion}

Les taurins Ndama du programme de sélection du CRZ-K constituent une petite population d'animaux qui a été préservée des brassages avec les races trypanosensibles. Ce programme a su malgré les contraintes, qu'il est possible d'améliorer les performances des animaux. Dans ce sens, les résultats de cette étude ont souligné que les taurins Ndama du CRZ-K de par leurs mensurations corporelles sont proches de la sous-population Grande Ndama dont la commercialisation génère plus de revenus. Ce progrès génétique pourrait encore s'accroitre avec un financement pérenne permettant la mise en œuvre efficiente du programme de sélection et la diffusion des géniteurs. La caractérisation moléculaire des animaux compléterait cette étude pour une meilleure connaissance des taurins Ndama du CRZ de Kolda.

\section{Remerciements}

Cette étude a été conduite dans le cadre du Projet d'Amélioration de la disponibilité et de la diffusion du matériel génétique amélioré des bovins Ndama et Gobra au Sénégal, financé par le Fonds National de Recherches Agricoles et Agro-alimentaires (FNRAA). Nous adressons nos sincères remerciements à ce partenaire financier. Nos remerciements vont également au Directeur et au personnel du Centre de Recherches Zootechniques de Kolda pour leur collaboration. 


\section{References:}

1. Aboagye, G. S. (2002). Phenotypic and genetic parameters in cattle populations in Ghana. AGTR Case Study. Nairobi, Kenya : ILRI.

2. Akouango, F., Ngokaka, C., Ewomango, P. et Kimbembe, E. (2010). Caractérisation morphométrique et reproductive des taureaux et vaches Ndama du Congo. Animal Genetic Ressources, 46 : 41- 47.

3. Badji, O. (1973). Institut Sénégalais de Recherches Agricoles ; Centre de Recherches Zootechniques de Kolda ; Rapport annuel de 1973. ISRA. - 24

4. Baldé, A. T. (2017). Caractérisation phénotypique du taurin Ndama du Sénégal. Mémoire de Master II en Biologie Animale, Université Cheikh Anta Diop, Dakar, 35.

5. Bosso, N. A. (2006). Genetic parameters for growth traits in N'Dama cattle under tsetse challenge in the Gambia. Ph.D. Thesis, Wageningen Institute of Animal Sciences, Wageningen, Pays-Bas. 147 p.

6. Camara, Y. (2012). Analyse génétique des performances zootechniques des bovins de race N'Dama et étude du système d'amélioration génétique à noyau ouvert. Diplôme de Master en Biotechnologies et Amélioration Génétique des Productions Agricoles, Institut Agronomique et Vétérinaire Hassan II, Maroc. 98.

7. Camara, Y., Ciss, M., Moula, N., Sissokho, M. M., Farnir, F. et Antoine-Moussiaux, N. (2019a). Determinants of breeders' participation to an indigenous cattle breeding program. Agronomy for Sustainable Development, 39: 44. https://doi.org/10.1007/s13593019-0591-1

8. Camara, Y., Sow, F., Govoeyi, B., Moula, N., Sissokho, M. M. et Antoine Moussiaux, N. (2019b). Stakeholder involvement in cattlebreeding program in developing $\mathrm{T}$ countries: A Delphi survey. Livestock Science, 228: 127-135. https://doi.org/10.1016/j.livsci.2019.08.014

9. Centre de Recherches Zootechniques de Kolda, Sénégal (1984) Rapport d'activité. In : Hoste, C. H., Chalon, E., D'Ieteren, G. et Trail, J. C. M. (1988). Le bétail trypanotolérant en Afrique occidentale et centrale- Bilan d'une décennie, Vol 3. Étude FAO Production et Santé Animales 20/3. Rome, 1988, 227.

10. Cornillon, P. A., Guyader, A., Husson, F., Jégou, N., Josse, J., Kloareg, M., Matzner-Løber, E. et Rouvière, L. (2010). Statistiques avec R. Rennes, Presses universitaires, 276 .

11. Coulibaly, T., Diall, L. et Outmani, A. (2014). Diagnostic de la situation de l'élevage Ndama dans son berceau de race (cercles de Bougouni et de Yanfolila). Ministère du Développement Rural, 
Direction Nationale des Productions et des Industries Animales, septembre 2014, Mali, 45p.

12. Coulomb, J. (1976). La race Ndama. Quelques caractéristiques zootechniques. Revue d'Elevage et de Médecine Vétérinaire des Pays Tropicaux, $29: 367-380$.

13. Diaïté, A. (1980). Contribution à l'étude des bovins trypanotolérants de la haute Casamance. Thèse : Médecine Vétérinaire : Dakar, 81.

14. Diack, F. (2009). Etude des systèmes d'élevage et Caractérisation morpho biométrique du taurin Ndama au sud du Sénégal. Mémoire de D.E.A en Biologie Animale, niversité Cheikh Anta Diop, Dakar, 58.

15. Dieye, P. N., Duteurtre, G., Sissokho, M. M., Sall, M. et Dia, D. (2003). "La production laitière périurbaine au sud du Sénégal. Saisonnalité de l'offre et performances économiques", Tropicultura, 21 (3) : 142-148.

16. Diop, M., Sissokho, M. M. et Niang, S. (1993). Mise en place d'un schéma de sélection à noyau ouvert pour l'amélioration génétique du taurin ndama : résultats du "screening" des vaches exceptionnelles dans le département de Kolda (Sénégal). Rapport d'avancement Avril 1993. ISRA/CRZ Kolda, 17.

17. Diouf, M. N., Seck, M. T., Diop, M., Sow, R. S., Seck, M., Sissoko, M., Diop, M., Fall, A. G., Skilton, R., Kemp, S., Njahira, M., Kyallo, M., Wanjala, B., Mbanjo, G., Kaduma, E., Nzuki, I., Wamonje, F. et Ndila, M. (2012). Caractérisation génétique des bovins Ndama au sud du Sénégal: résultats préliminaires.

18. Doutressoule (1947). L'élevage en Afrique occidentale française, Éditions Larose, Paris. 300 p.

19. Epstein, H. (1971). The origin of the domestic animals of Africa: Africana Publishing Corporation.

20. Fall, A., Diop, M., Sandford, J., Wissocq, Y. J., Durkin, J. et Trail, J. C. M. (1982). Evaluation des productivités des ovins Djallonke et des taurins Ndama au CRZ de Kolda, Sénégal. CIPEA, Rapport de Rechrche $\mathrm{N}^{\circ}$ 3, Addis Abeba (Ethiopie), 74.

21. FAO (1985). Programme international de coordination du développement laitier et programme international de développement du secteur des viandes. Projet de rapport : République de la Guinée Bissau. FAO, Rome.

22. FAO (2012). Phenotypic characterization of animal genetic resources. FAO Animal Production and Health Guidelines No. 11. Rome, 158.

23. Ganyo, E. Y., Boampong, J. N., Masiga, D. K., Villinger, J. et Turkson, P. K. (2018). Haematology of N'Dama and West African Shorthorn cattle herds under natural Trypanosoma vivax challenge in Ghana 
[version 2; referees: 2 approved, 1 approved with reservations]. F1000Research, 7: 314.

24. Gueye, E. H., Nicolas, A, Diao, B., Ndiaye, L., Faye, O. et Kandé, M. (1979). Identification des taurins ndama en milieu rural. Etude de la composition de la structure et de la couleur des robes des troupeaux taurins. Rapport d'activités, Juillet 1979, 27.

25. Gueye, E. H., Boye, C. M., Bayo, M. et Sané, I. (1980). Prétestage des taurillons au CRZ de Kolda, lot V, rapport d'activités, Juillet 1980, 29.

26. Kamga-Waladjo, A. R., Thiam, O., Sultan, J. et Diop P. E. H. (2005). Evaluation des Performances des Ndama et des produits de l'insémination artificielle en République de Guinée. RASPA, 3 (2) : 93-97.

27. Khang'Mate, A., Lahlou-Kassi, A., Bakana, B. et Kahungu, M. (2000). Performances de reproduction des bovins Ndama dans le diocèse d'Idiofa au Congo. Revue de Médecine Vétérinaire, 151 (6) : 511-516.

28. Laveissière, C. et Toure, S. (1982). La répartition des glossines au Sénégal : carte à $1 / 2000000$.

29. Mackinnon, M. J., Meyer, K., and Hetzel, D. J. S. (1991). Genetic variation and covariation for growth, parasite resistance and heat tolerance in tropical cattle. Livestock Production Science 27: 105-122.

30. MEPA (Ministère de l'Elevage et de la Production Animale), 2007 : Rapport Enquête sur la Productivité des Troupeaux Bovins : estimation des productions de viande et de lait. République du Sénégal, Novembre 2007.

31. Ndiaye, N. P. (2015). Evaluation de la diversité et de la structure génétique des races bovines locales (Artiodactyla : bovidea) du Sénégal. Thèse de doctorat unique, université Cheikh Anta Diop, Dakar, 215.

32. Ndiaye, N. P., Sawadogo, G. J. et Sembene, P. M. (2015). Race Bovines Locales (Artiodactyla : Bovidae) du Sénégal - Diversités phénotypique et génétique. Editions Universitaires Européenes.

33. N’goran, K. E., Bamba, K. L., Kouassi, N. C., Loukou, N. E., Dayo, G-K., Sangare, M. et Yapi-Gnaore C. V. (2018). Multivariate Analysis for Morphological Characteristics of N'dama Cattle Breed in Two Agro-ecological Zones of Côte d'Ivoire. European Scientific Journal, $14(3): 602-621$.

34. Okouyi, M. W. M., Kamga-Waladjo, A. R., Diarra, S. et Hanzen, Ch. (2014). Caractéristiques de reproduction de la femelle trypanotolérante de race N'Dama. RASPA, 12 (1) : 1-7.

35. Pagot, J. et Delaine, R. (1959). Étude biométrique de la croissance des taurins N'dama. Revue d'Elevage et de Médecine Vétérinaire des Pays Tropicaux, 12 (4) : 405-416. 
36. Planchenault, D., Tall, S. H. et Traoré, M. T. (1984). Amélioration génétique des bovins N'Dama. Etudes en milieu extensif au Mali_ I. Caractéristiques du bétail N'Dama au ranch de Madina-Diassa. Revue d'Elevage et de Médecine Vétérinaire des Pays Tropicaux, 37 (4) : 488495.

37. PROGEBE ILRI (2011). Revue des politiques d'élevage en rapport avec la gestion durable du bétail ruminant endémique. Sénégal, Février 2011, 64.

38. R Core Team (2016). R: A language and environment for statistical computing. R Foundation for Statistical Computing, Vienna, Austria. URL http://www.R-project.org/.

39. Sambe, B. S., Diouf, M. N., Ciss, M., Baldé, A. T., Badji, M.N., Diop, M. and Sembène, M. (2019). Phenotypic characterization of gobra zebu cattle of centre de recherches zootechniques of dahra. International Journal of Advanced Research, 7 (6), 26-34

40. Sauveroche, B. et Wagner, H. G. (1993). Physiologie de la reproduction des bovins trypanotolérants : Synthèses des connaissances actuelles. FAO. ed., Rome, 149.

41. Sissokho, M. M., Diatta, A. et Camara, Y. (2010). Institut Sénégalais de Recherches Agricoles, Centre de Recherches Zootechniques de Kolda, Rapport technique annuel d'activités de 2009, 96p.

42. Sokouri, D. P., Loukou, N. E., Yapi-Gnaore, C. V., Mondeil, F. et Gnangbe, F. (2007). Caractérisation phénotypique des bovins à viande (Bos taurus et Bos indicus) au centre (Bouaké) et au nord (Korhogo) de la Côte d'Ivoire. Animal Genetic Resources information, 40, 43-53.

43. Sokouri, D. P., Gbodjo, Z. L., N'Goran, K. E. et Soro, B. (2014). Performances de reproduction et production laitière de croisés Montbéliarde x N'Dama du "Projet Laitier Sud' (Côte d'Ivoire). International Journal of Biological and Chemical Sciences, 8 (3): 925 936.

44. Touré, S. (1971). Les glossines (Diptera, Glossinidae) du Sénégal : écologie, répartition géographique et incidence sur les trypanosomes. Revue d'Elevage et de Médecine Vétérinaire des Pays Tropicaux, 24(4), 551-563.

45. Touré, S. M. (1977). La trypanotolérance Revue de connaissances. Revue d'Elevage et de Médecine Vétérinaire des Pays Tropicaux, 30 (2), 157-174.

46. Wane, M., Diouf, M. N., Diop, M. et Kamga Waladjo, A. R. (2017). Revue de programme de sélection bovine : cas du taurin Ndama au Centre de Recherches Zootechniques de Kolda (Sénégal). Institut sénégalais de Recherches agricoles (ISRA), 61. 